\title{
Investigation of the Crystallization Kinetics in Ge-Sb-Te-Bi Thin Films for Phase Change Memory Application
}

\author{
A.V. Babich ${ }^{a, *}$, A.A. Sherchenkov ${ }^{a}$, S.A. Kozyukhin ${ }^{b}$ And S.P. Timoshenkov ${ }^{a}$ \\ ${ }^{a}$ National Research University of Electronic Technology, \\ Building 1, Shokin square, Zelenograd, Moscow, Russian Federation \\ ${ }^{b}$ Kurnakov Institute of General and Inorganic Chemistry of the Russian Academy of Sciences, \\ 31 Leninsky prospect, Moscow, Russian Federation
}

\begin{abstract}
In this work the mechanism and kinetics of crystallization of the $\mathrm{Ge}_{2} \mathrm{Sb}_{2} \mathrm{Te}_{5}+\mathrm{Bi}$ thin films were investigated using differential scanning calorimetry. $\mathrm{Ge}_{2} \mathrm{Sb}_{2} \mathrm{Te}_{5}$ with different amounts of $\mathrm{Bi}(0,0.2,0.5,0.8,1,3,5$ wt.\%) was synthesized using quenching technique. Thin films were prepared by thermal evaporation of synthesized materials. $\mathrm{X}$-ray diffraction has shown that synthesized materials had trigonal modification of $\mathrm{Ge}_{2} \mathrm{Sb}_{2} \mathrm{Te}_{5}$. Introduction of $\mathrm{Bi}$ led to the appearance of trigonal modification of $\mathrm{Bi}_{2} \mathrm{Ge}_{2} \mathrm{Te}_{5}$, which indicates on the replacement of $\mathrm{Sb}$ by $\mathrm{Bi}$. Asdeposited thin films were amorphous up to $3 \%$ of $\mathrm{Bi}$. Higher concentrations of $\mathrm{Bi}$ led to the appearance of crystalline phases. Composition of thin films was verified by Rutherford backscattering, and was found to be close to that of the synthesized materials. The joint application of model-free Ozawa-Flynn-Wall and model-fitting Coates-Redfern methods allowed to estimate kinetic triplet for crystallization process of GST225+Bi thin films, and to predict data processing and storage times of the phase change memory cells. It was shown that GST $225+0.5$ wt. $\%$ Bi thin films have the most promising kinetic characteristics among the investigated materials, due to the predicted smallest data processing and largest storage times.
\end{abstract}

DOI: 10.12693/APhysPolA.129.717

PACS/topics: $65.60 .+\mathrm{a}$

\section{Introduction}

Currently an active development of non-volatile memory devices is carried out. Phase change memory (PCM) is considered to be one of the most promising candidates for the next generation of non-volatile memory devices.

Concept of phase-change memory was proposed by Stanford Ovshinsky in 1968 [1]. He has demonstrated the possibility of electrical and optical ways of storing information by using devices on the basis of chalcogenide glassy semiconductors [2,3]. However, nearly 30 years passed from the first generation of PCM without exciting parameters, till the appearance of commercially successful disks of different formats, such as DVD, and the last generation - the Blu-Ray discs $[4,5]$.

Nowadays two main types of PCM memory devices exist, optical and electrical phase-change memories. The working principle of both types of devices is based on the rapid reversible amorphous-to-crystalline phase transitions, which can be initiated by laser or electrical pulses, respectively. PCM materials in amorphous and crystalline states have very different optical and electrical properties, and are attributed the "zero" and "one" logical states.

At the dawn of PCM technology the main problems were connected with the slow crystallization rate, limited cyclability, and spontaneous crystallization at room

*corresponding author; e-mail: drent@yandex.ru temperature. A breakthrough was reached with the discovery of fast switching stable compositions on the quasibinary line between GeTe and $\mathrm{Sb}_{2} \mathrm{Te}_{3}$ by Yamada and coauthors in 1987 [6]. This discovery led to the commercial success of CD, DVD, and Blu-ray disks.

Three compounds exist on this quasi-binary line: $\mathrm{Ge}_{2} \mathrm{Sb}_{2} \mathrm{Te}_{5}$ (GST225), $\mathrm{GeSb}_{2} \mathrm{Te}_{4} \quad$ (GST124), and $\mathrm{GeSb}_{4} \mathrm{Te}_{7}$ (GST147). These compounds form homologous series and have similar phase diagrams [7]. They crystallize in the metastable $\mathrm{NaCl}$ type structure, which is highly symmetric and isotropic $[8,9]$. Large oscillations of atoms [7] and high concentration of vacancies [10-12] cause disordering of the structure, which becomes close to amorphous, to a large extent. In this case atoms do not need large displacements and diffusion to take their places in the crystal structure, which explains rapid crystallization [13]. Small difference of the volumes of amorphous and crystalline phases leads to small stresses due to the phase transition $[7,14]$. Together with the absence of phase separation this characteristic feature determines high cyclability of the materials [7]. From these points of view the alloys on the quasi-binary line of GeTe- $\mathrm{Sb}_{2} \mathrm{Te}_{3}$ are the most perspective PCM materials, and the most promising among them is considered to be GST225 due to the complex of properties (appreciable stability at room temperature, high crystallization rate and good reversibility between amorphous and crystalline phases).

Noticeable progress was achieved in recent years in the development of PCM. However, despite the success, the electrical PCM does not become a widespread technology. 
This is explained by the existence of quite specific requirements to the phase-change materials. Some of these requirements are contradictory and quite difficult to be achieved, which greatly complicates the improvement of the technology. In this case, improving of PCM technology requires effective methods for controlling the properties of PCM materials, and possibility to predict the behavior of the materials at different temperatures.

Insensitivity of most chalcogenide glasses to doping complicates the problem. However, previously [15] we have shown that doping with $\mathrm{Bi}$ allows modification of thermal properties of GST225. Selection of the dopant is based on the assumption that doping by the isomorphic element with slightly different atomic radius from that of the antimony will go by the replacement mechanism, and will introduce minimum deformations and mechanical stresses in the material matrix. Besides, it is possible to modify the biding energy by the replacing of $\mathrm{Sb}-\mathrm{Te}$ bonds by the $\mathrm{Bi}-\mathrm{Te}$, and so to vary the thermal properties.

In addition to the modification of thermal properties such doping can affect the crystallization process, which determine the data processing rate of the PCM. Thus, the aim of this work is to study mechanism and kinetics of crystallization for thin films of GST225, and the influence of Bi doping on them.

\section{Experimental method}

GST225 with different amounts of $\mathrm{Bi}(0,0.2,0.5,0.8$, $1,3,5$ wt.\%) was synthesized using quenching technique, which was proposed in [16], and modified in [17]. Thin films were prepared by thermal evaporation of synthesized materials. Substrate temperature during the deposition had not exceeded $50^{\circ} \mathrm{C}$. Pressure in the chamber was $10^{-4} \mathrm{~Pa}$.

X-ray diffraction (Rigaku D/MAX, $\mathrm{Cu} \mathrm{K}_{\alpha}, \lambda=$ $0.15481 \mathrm{~nm}$ ) has shown that the synthesized materials had trigonal modification of $\mathrm{Ge}_{2} \mathrm{Sb}_{2} \mathrm{Te}_{5}$. Introduction of $\mathrm{Bi}$ led to the appearance of trigonal modification of $\mathrm{Bi}_{2} \mathrm{Ge}_{2} \mathrm{Te}_{5}$, which indicated on the replacement of $\mathrm{Sb}$ by $\mathrm{Bi}$. As-deposited thin films were amorphous up to $3 \%$ of $\mathrm{Bi}$. Higher concentrations of $\mathrm{Bi}$ led to the appearance of crystalline phases.

Compositions of thin films were verified by Rutherford backscattering $\left(E_{d}=1.0, E_{\alpha}=2.7 \mathrm{MeV}, 135^{\circ}\right.$ scattering angle). It was shown that compositions of thin films were close to those of the synthesized materials [15].

Differential scanning calorimetry (DSC-50, Shimadzu) was used for the investigation of thermal properties. Measurements were carried out at eight different heating rates (from 1 to $90^{\circ} \mathrm{C} / \mathrm{min}$ ) in a nitrogen flow $(20 \mathrm{ml} / \mathrm{min})$.

To determine the kinetic triplet for crystallization process of GST thin films we have used the isoconversional model-free method of Ozawa-Flynn-Wall (OFW) [18], and Coates-Redfern model-fitting method [18]. Such joint use of model-free and model-fitting methods allowed us to estimate activation energy and pre-exponential factor as functions of conversion, and to determine the reaction model.

\section{Results and discussion}

Differential scanning calorimetry of the as-deposited amorphous films revealed a number of heat effects with crystallization peaks for all compositions laying in the temperature range of $135-220^{\circ} \mathrm{C}$ (Fig. 1).

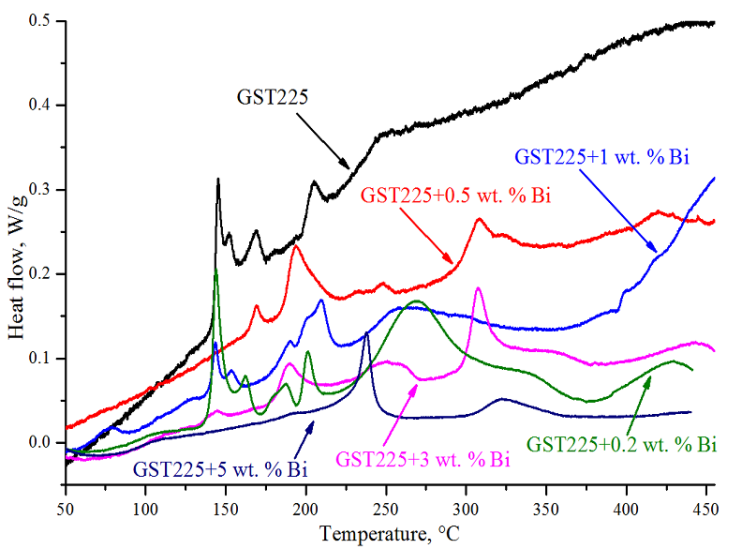

Fig. 1. DSC scans of as-deposited amorphous thin films of $\mathrm{Ge}_{2} \mathrm{Sb}_{2} \mathrm{Te}_{5}$ with different Bi contents for heating rate of $10^{\circ} \mathrm{C} / \mathrm{min}$.

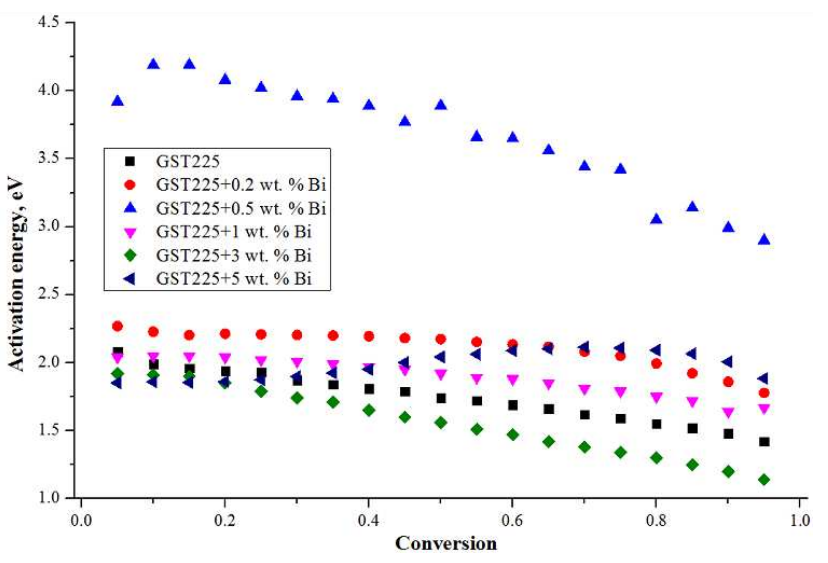

Fig. 2. Effective activation energy of crystallization for compositions with Bi.

It was found that for investigated thin films, with the exception of $\mathrm{Ge}_{2} \mathrm{Sb}_{2} \mathrm{Te}_{5}+5$ wt. $\%$ Bi the effective activation energy estimated by Ozawa-Flynn-Wall method gradually decreases with the increase of conversion (Fig. 2). This result indicates that crystallization of GST thin film is a complex process, and is determined by two parallel processes, the nucleation and the crystalline growth. Contribution of the first process gradually decreases, while that of the second increases. Sufficiently higher values of the effective activation energy in comparison with other investigated materials were established for $\mathrm{Ge}_{2} \mathrm{Sb}_{2} \mathrm{Te}_{5}+0.5 \mathrm{wt} . \% \mathrm{Bi}$ thin films. 


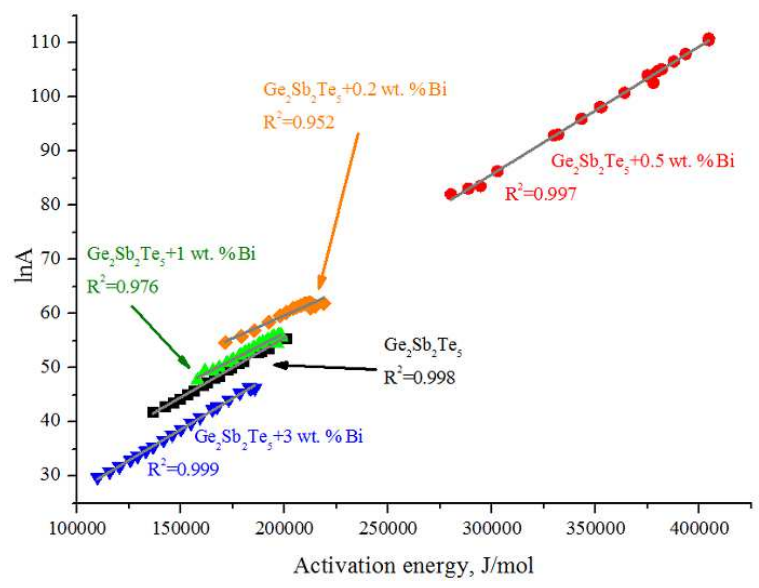

Fig. 3. Compensation effect for GST225 with different Bi content.

The use of effective activation energies $\left(E_{a}\right)$ obtained by the OFW method allowed us to estimate preexponential factor $A$ as a function of conversion. The observed correlation between the pre-exponential factor $A$ and effective activation energy $E_{a}$ (Fig. 3) corresponds to the compensation effect, which is expressed by the equation [19]:

$$
\ln A=a E_{a}+b,
$$

where $a$ and $b$ are constants.

This effect reflects the relation according to which the decrease in reaction rate due to the increase in activation energy is compensated by the increase in the magnitude of $\ln A$.

The use of Coates - Redfern model-fitting method showed that for all investigated compositions the most probable models describing crystallization process of thin films are the second and third order reaction models (Table I). These models assume the spontaneous growth of the nuclei without overlapping, so they provide an adequate description of the reaction till the beginning of the overlapping of nuclei [19].

TABLE I

Crystallization models for GST225+Bi thin films.

\begin{tabular}{c|c|c|c}
\hline \hline Compositions & $\begin{array}{c}\text { Reaction } \\
\text { model }\end{array}$ & $\begin{array}{c}\text { Differential } \\
\text { form, } \mathrm{f}(\alpha)\end{array}$ & $\begin{array}{c}\text { Integral } \\
\text { form, } \mathrm{g}(\alpha)\end{array}$ \\
\hline GST 225 & 2nd-order & $(1-\alpha)^{2}$ & {$[1 /(1-\alpha)]-1$} \\
GST 225+0.2 wt.\% Bi & 3rd-order & $(1-\alpha)^{3}$ & {$\left[1 /(1-\alpha)^{2}\right]-1$} \\
GST 225+0.5 wt.\% Bi & 2nd-order & $(1-\alpha)^{2}$ & {$[1 /(1-\alpha)]-1$} \\
GST 225+1 wt.\% Bi & 2nd-order & $(1-\alpha)^{2}$ & {$[1 /(1-\alpha)]-1$} \\
GST 225+3 wt.\% Bi & 3rd-order & $(1-\alpha)^{3}$ & {$\left[1 /(1-\alpha)^{2}\right]-1$} \\
GST 225+5 wt.\% Bi & 2nd-order & $(1-\alpha)^{2}$ & {$[1 /(1-\alpha)]-1$}
\end{tabular}

Then we have used the determined kinetic triplet for the estimation of possible data processing and storage times of the PCM cell based on investigated materials. We have assumed that logical "zero" and "one" states differ by the values of conductivity not less, than by one order of magnitude. Taking into consideration that crystallization of our films is accompanied by a drop of resistivity by 3-4 orders of magnitude, we estimated conversion $\alpha$ (crystalline fraction), which corresponds to the transition between the " 0 " and " 1 " logical states of the PCM cell by the equation [20]

$$
\alpha=\left(\ln \sigma-\ln \sigma_{\mathrm{a}}\right) /\left(\ln \sigma_{\mathrm{c}}-\ln \sigma_{\mathrm{a}}\right),
$$

where $\sigma_{\mathrm{a}}$ is the conductivity of a completely amorphous material, $\sigma_{\mathrm{c}}$ is the conductivity of a completely crystalline material, and $\sigma$ is the conductivity of the material with the crystalline fraction $\alpha$.

The calculations have shown that the transition between " 0 " and "1" occurs at $\alpha=0.3$. By using this value of conversion we have estimated the possible data processing and storage times of PCM cells as transition time between amorphous ("0") and crystalline ("1") states of the films based of investigated materials for $\alpha=0.3$ at different temperatures which can be calculated by the equation [19]:

$$
t=g(\alpha) /\left[A \exp \left(E_{a} /(R T)\right)\right]
$$

Calculated dependencies of transition times for $\alpha=0.3$ on crystallization temperatures for all investigated materials are shown in Fig. 4.

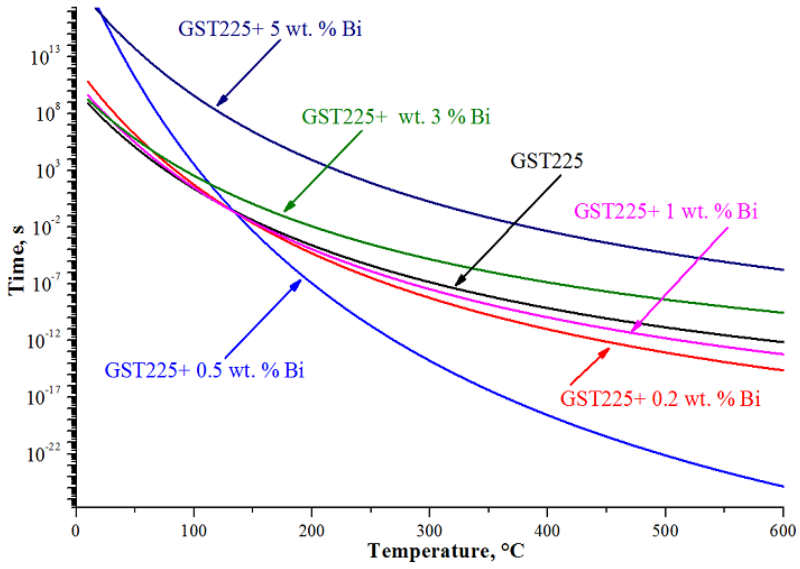

Fig. 4. Estimation of transition time for GST225 with different $\mathrm{Bi}$ content.

Sufficient difference is observed for GST with $0.5 \%$ of $\mathrm{Bi}$. It has the least transition time, which at $250^{\circ} \mathrm{C}$ has an extremely low value (approximately $2 \times 10^{-11} \mathrm{~s}$ ). However, even smaller times can be calculated for the higher crystallization temperatures, which is quite doubtful. Important role in the crystallization process of GST thin films plays the initial period of nucleation. In this case, calculations must have a lower limit. This limit is determined by the time of nucleation period, because during this period the nucleation, but not crystalline growth, plays the dominating role. For chalcogenide materials this period can be from tens to hundreds of ns.

The largest storage time at room temperature also has GST with $0.5 \%$ of $\mathrm{Bi}$. This result can be explained by the highest value of activation energy of crystallization, 
which provides greater sensitivity of crystallization rate to the temperature, and large range of crystallization rates between the low and high temperatures.

\section{Conclusions}

Thus, in this work the mechanism and kinetics of crystallization for GST225+Bi thin films were investigated using differential scanning calorimetry. The joint application of model-free Ozawa-Flynn-Wall and modelfitting Coates-Redfern methods allowed to estimate kinetic triplet for crystallization process of GST225+Bi thin films, i.e. the effective activation energies and preexponential factors as the functions of conversion, and to establish the crystallization models. The obtained kinetic triplet permits one to describe crystallization of GST225+Bi films, and predict data processing and storage times of the phase change memory cells. It was shown that GST225+0.5 wt.\% Bi thin films have the most promising kinetic characteristics among the investigated materials due to the predicted smallest data processing and largest storage times.

\section{Acknowledgments}

This study was supported by Ministry of Education and Science of RF (project ID: RFMEFI57814X0085).

\section{References}

[1] S.R. Ovshinsky, Phys. Rev. Lett. 21, 1450 (1968).

[2] S.R. Ovshinsky, H. Fritzsche, Metall. Trans. 2, 641 (1971).

[3] J. Feileib, S. Iwasa, S.C. Moss, J.P. Neufville, S.R. Ovchinsky, J. Non-Cryst. Solids 8-10, 909 (1972).

[4] E.R.Meinders, A.V. Mijiritskii, L. van Pieterson, M. Wuttig, Optical Data Storage Phase-Change Media and Recording, Philips Research Book Series, V. 4, Springer-Verlag, Berlin 2006.

[5] T. Narahara, S. Kobayashi, M. Hattori, Y. Shimpuku, G. van den Enden, J. Kahlman, M. van Dijk, R. van Woudenberg, Jpn. J. Appl. Phys 39, 912 (2000).
[6] N. Yamada, E. Ohno, N. Akahira, K. Nishiuchi, K. Nagata, M. Takao, Jpn. J. Appl. Phys. 26, 61 (1987).

[7] S. Raoux, M. Wuttig, Phase Change Materials, Springer, New York 2009

[8] A.V. Kolobov, P. Fons, J. Tominaga, A.I. Frenkel, A.L. Ankudinov, S.N. Yannopoulos, K.S. Andrikopoulos, T. Uruga, Jpn. J. Appl. Phys. 44 3345 (2005).

[9] N. Yamada, MRS Bull. 21, 48 (1996).

[10] M. Wuttig, D.L. Sebkink, D. Wamwangi, W. Wełnic, M. Gilleßen, R. Dronskowski, Nature Mater. 6, 122 (2007).

[11] A.V. Kolobov, P. Fons, J. Tominaga, S. Ovshinsky, Phys. Rev. B 87, 165206 (2013).

[12] T.H. Lee, S.R. Elliott, Phys. Rev. B 84, 094124 (2011).

[13] J.J. Gervacio Arciniega, E. Prokhorov, F. Espinoza Beltran, G. Trapaga, Crystallization - Science and Technology, Crystallization of Ge:Sb:Te Thin Films for Phase Change Memory Application, Chap. 16, InTech, Croatia 2012.

[14] K.N. Chen, L. Krusin-Elbaum, C. Cabral Jr., C. Lavoie, J. Sun, S. Rossnagel, Non-Volatile Semiconductor Memory Workshop (2006), IEEE, 2006.

[15] A. Sherchenkov, S. Kozyukhin, A. Babich, P. Lazarenko. J. Non-Cryst. Solids 377, 26 (2013).

[16] N.Kh. Abrikosov, G.T. Danilova-Dobryakova, Izv. Akad. Nauk SSSR, Neorg. Mater. 1, 204 (1965).

[17] S.A. Kozyukhin, A.A. Sherchenkov, E.V. Gorschkova, V. Kudoyarova, A. Vargunin, Phys. Status Solidi C 7, 848 (2010).

[18] S. Kozyukhin, A. Sherchenkov, A. Babich, P. Lazarenko, Huy Phuc Nguyen, O. Prikhodko, Canad. J. Phys. 92, 684 (2014).

[19] M.E. Brown, Handbook of thermal analysis and Calorimetry, Elsiever Science B.V., Amsterdam 1998.

[20] N. Mehta, A. Kumar, J. Therm. Anal. Calorim. 83, 669 (2006). 